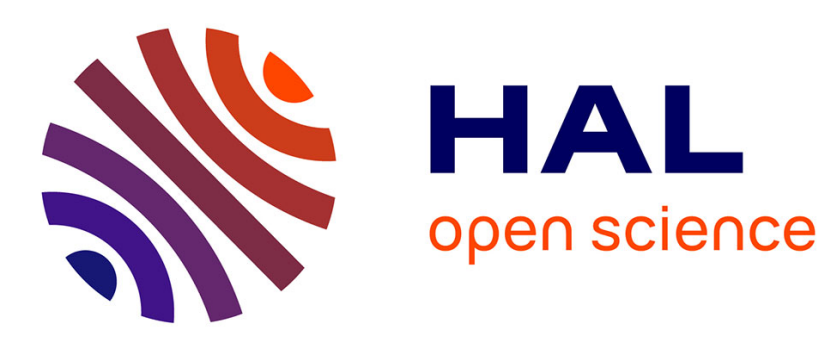

\title{
Solute friction and forest interaction
}

Ghiath Monnet, Benoit Devincre

\section{To cite this version:}

Ghiath Monnet, Benoit Devincre. Solute friction and forest interaction. Philosophical Magazine, 2006, 86 (11), pp.1555-1565. 10.1080/14786430500398425 . hal-00513632

\section{HAL Id: hal-00513632 \\ https://hal.science/hal-00513632}

Submitted on 1 Sep 2010

HAL is a multi-disciplinary open access archive for the deposit and dissemination of scientific research documents, whether they are published or not. The documents may come from teaching and research institutions in France or abroad, or from public or private research centers.
L'archive ouverte pluridisciplinaire HAL, est destinée au dépôt et à la diffusion de documents scientifiques de niveau recherche, publiés ou non, émanant des établissements d'enseignement et de recherche français ou étrangers, des laboratoires publics ou privés. 


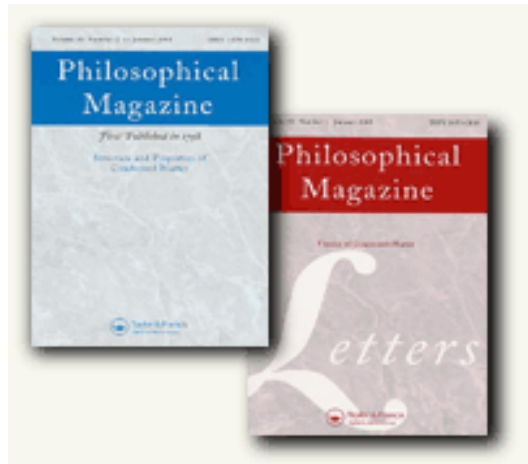

\section{Solute friction and forest interaction}

\begin{tabular}{|r|l|}
\hline Journal: & Philosophical Magazine \& Philosophical Magazine Letters \\
\hline Manuscript ID: & TPHM-05-Aug-0360.R1 \\
\hline Journal Selection: & Philosophical Magazine \\
\hline Date Submitted by the \\
Author: & 30 -Sep-2005 \\
\hline Complete List of Authors: & $\begin{array}{l}\text { Monnet, Ghiath; Electricité de France, MMC } \\
\text { Devincre, Benoit; LEM-ONERA-CNRS }\end{array}$ \\
\hline Keywords: & solid solutions, friction, dislocations, dislocation dynamics \\
\hline Keywords (user supplied): & Forest hardening, dislocation interaction, alloy friction \\
\hline &
\end{tabular}

\section{s ScholarONE" \\ Manuscript Central}




\section{Solute friction and forest interaction}

7

8

\section{Abstract}

Solute friction is known to prevail in crystals where a solution of point defects results in a diffuse resistance to dislocation motion. This property is often used to strengthen materials. In this paper we show that it affects also dislocation-dislocation interactions. Dislocation dynamics simulations are used to investigate and quantify this property. The solute friction results in a shielding of elastic interactions leading to a significant decrease of the intrinsic strengths of junction and annihilation reactions. Simulations in static and dynamic conditions show that the interaction stability decreases with the friction stress. A model is proposed to account for the modification of the interaction coefficient predicted by massive simulations in latent hardening conditions. Results suggest that the observed softening is mainly due to the decrease of the line tension of dislocations involved in the dislocation-dislocation interactions.

Keywrods: Forest hardening, solid solution, dislocation dynamics, alloy friction 


\section{Introduction}

Solid solution friction on dislocations is a property common to many alloys [1]. In materials such as BCC and HCP crystals, solid solution hardening may reduce dislocation mobility and therefore can tremendously increase the material flow stress. At low temperatures, alloy friction is a complex phenomenon that can hardly be distinguished from lattice friction since both mechanisms restrict screw dislocation mobility resulting in a complex thermally activated behavior. At higher temperature, when lattice friction is almost completely overcome by thermal activation, an athermal friction stress, $\tau_{F}$, remains. For well-annealed samples this friction stress is constant and contributes to a large part of the Critical Resolved Shear Stress (CRSS) [2-3].

In the presence of alloy friction, forest hardening is assumed to take place and the strength of dislocation-dislocation interactions is generally assumed to be those observed in the pure material. The critical stress for plastic flow, $\tau_{c}$, is conventionally defined as:

$$
\tau_{c}=\tau_{F}+\alpha \mu b \sqrt{\rho_{f}}
$$

where, $\mu$ is the shear modulus, $b$ is the Burgers vector and $\rho_{f}$ is the forest dislocation density, close to the total density in many instances. The forest interaction coefficient, $\alpha$, is temperature independent, and is a measure of the mean strength of dislocation-dislocation interactions during plastic deformation [4-5].

In this paper, it is shown that under a large alloys friction $\tau_{F}$, the stability of forest reactions is decreased and this is manifested macroscopically by a decrease of $\alpha$. In order to explain this phenomenon, the stability of forest reactions between isolated dislocations is firstly 
investigated. Then, the influence of $\tau_{F}$ on the CRSS is measured with the help of Dislocation Dynamics (DD) simulations.

\section{Elementary dislocation reactions under high alloy friction}

To illustrate the effect of a constant friction stress on dislocation-dislocation reactions, we first consider the case of an isolated junction of finite size between two dislocations under zero applied stress. $\mathbf{b}_{\mathrm{i}}$ and $\mathbf{l}_{\mathbf{i}}$ define the dislocation Burgers vector and the line direction, respectively. Contact reaction occurs along the axis, $\mathbf{I}_{\mathrm{j}}$, at the intersection of the two glide planes. Depending on the relation between $\mathbf{b}_{1}$ and $\mathbf{b}_{2}$, elastic interactions can lead to several types of configurations [6]. To simplify the mathematics, we present the case of a symmetrical junction between two initially edge dislocations, belonging to two different slip planes, of same type of Burgers vector and making the same angle with respect to $\mathbf{I}_{\mathbf{j}}$. This configuration is schematically illustrated on figure 1 . In this figure, dislocation 1 (bold black line) and its Burgers vector do not belong to the same plane as dislocation 2 (thin gray line). The configuration is of course $3 \mathrm{D}$, but one of the planes was rotated to match the second one in order to draw the $2 \mathrm{D}$ scheme of the figure.

However, the conclusion is quite general and independent of the initial configuration.

(Figure 1)

When $\tau_{F}$ is small or equals zero, the zipping of junctions and the equilibrium shape is mostly controlled by line tension [6-7]. Therefore, the dislocations connected at the junction triple nodes never exhibit a significant curvature and the relaxed state can be predicted by means of simple formulations [8-10]. Conversely under a large $\tau_{F}$, different expressions are needed to
Deleted: of finite size

Deleted: different

Deleted: two edge dislocations 
account for the significant modifications observed in the relaxed states. For reason of symmetry, in figure 1 only one fourth of the junction is drawn. $X_{1}$ denotes one half of the junction length and the curved line represents one of the two dislocation arms involved in the junction reaction.

Initially the two dislocations each make an angle $\beta$ with the junction direction. In the relaxed state, every dislocation arm makes an angle $\varphi$ with its Burgers vectors $(\varphi=\pi / 2$ before reaction). Assuming a junction is formed, the configuration when relaxed must fulfil two conditions:

(i) Far from the triple node, the dislocation remains in its initial straight direction. This is because in this region, dislocation-dislocation interactions are weak and the gliding forces cannot overcome $\tau_{F}$.

(ii) At the junction triple node, the balance of line tensions imposes the angle $\theta$ between the junction direction and the connected dislocation lines.

As a consequence, junction zipping induces a bow out in the intermediate region. The radius of curvature, $R$, increases during the zipping up to the equilibrium value roughly equals $\mu b / \tau_{F}$. Indeed, during the zipping of the junction, the lengths of the curved part of the dislocation arm increases, which increases the friction on this part. The equilibrium shape is then reached when the driving force for the zipping of the junction, i.e. the decrease of energy induced by the junction formation, is balanced by the stress necessary to acquire such curvature under a given friction stress, i.e. $\mu b / \tau_{F}$.

To calculate precisely the junction length, $X=2 X_{1}$, it is necessary to account for the character dependence of the line tension in the vicinity of the junction triple nodes [8-10]. For the sake of simplicity, the expression "character angle" is used to designate the angle between the direction of the dislocation line and the direction of its Burgers vector. The condition of equilibrium at triple nodes is: 


$$
b_{j}^{2}\left(1-v \cos ^{2} \alpha_{j}\right)=2 \cos \theta b^{2}\left(1-v \cos ^{2} \varphi\right)
$$

where $b_{j}$ is the modulus of the junction Burgers vector, $\alpha_{j}$ the character angle of the junction, $v$ is the Poisson's ratio and $\varphi$ the character angle of the junction arms, i.e. the two dislocation lines connected at the triple node. According to figure $1, \varphi=\pi / 2+\beta-\theta$, where $\beta$ is a given angle between the junction direction and the direction of the initial dislocation line. The latter equation combined to equation (2) allows us to calculate $\theta$ and $\varphi$. On the other hand, from figure 1 and using elementary mathematics, we can derive equation 3 giving the junction length:

$$
X=2 \frac{\sin (\theta-\beta)}{\sin (\beta)} \tan \left(\frac{\theta-\beta}{2}\right) \frac{\mu b}{\tau_{F}}
$$

From equation 3 , it appears that the junction length should scale with $\mu b / \tau_{F}$, i.e. with the dislocation radius of curvature close to triple nodes.

To validate this result, DD simulations were used to precisely calculate the variation of $\mathrm{X}$ with different values of $\tau_{F}$. Readers interested by a description of the DD simulation are invited to consult [11-12]. In order to account for the friction stress in DD simulations, a simple linear form of dislocations mobility law, i.e. $V=B\left(\tau_{e f f}-\tau_{F}\right)$, is used for all investigated systems. $\tau_{\text {eff }}$ is the effective stress on the dislocation segment and $B$ a viscous coefficient accounting for phonon drag in the solid. By definition, when $\left|\tau_{e f f}\right|<\tau_{F}, V$ equals zero. It is worth noting that alternative calculations using more realistic dislocation mobility forms, like in [12-13], do not change the conclusions of the present work. 
As summarized in figure 2, the agreement between the results of DD simulations and the prediction of equation 3 is very good. Some discrepancy is observed only for large values of $\mu b / \tau_{F}$ since then the dislocation portions exhibiting a curvature reach the pinned end of the dislocations.

(Figure 2)

To estimate the stress, $\Delta \tau=\left(\tau_{c}-\tau_{F}\right)$, necessary for the breakaway of a mobile dislocation intersecting a forest obstacle, it is interesting to consider the equilibrium shapes of dislocation-dislocation interactions when the applied stress $\tau_{a p p}$ equals $\tau_{F}$. For simplicity, we focus on the case of collinear annihilations to clearly illustrate the alloy friction effect. This reaction is also affected by the friction stress and it exhibits the same tendency expressed in equation 3. Above all, it is by far the strongest reaction that takes place during plastic deformation [14].

Depending on loading conditions, an applied stress modifies the relaxed configurations under multiple combinations, depending on the Schmid factor on the involved systems. In all cases though, the applied stress induces an asymmetry in the dislocation configurations since it has opposite effects on each side of the reaction by-products. In the following, the dislocation shapes obtained when the Schmid factor is zero on one of the two intersecting slip planes. This case is referred to as a latent configuration. This is a simple situation to analyse and it is also the most frequent. It corresponds to the general situation when a mobile dislocation intersects an immobile forest obstacle with $\tau_{\text {eff }} \approx 0$.

$-6-$ 
From figure 3 one can find out that $X_{1}$ is geometrically related to $\theta_{1}$ according to

$$
\pi-\beta-\operatorname{Arccos}\left(1-X_{1} \frac{\tau_{F}}{\mu b} \sin \beta\right)=\operatorname{Arctan} \frac{L_{o} \sin \beta}{L_{o} \cos (\beta)-X_{1}}
$$

where $L_{\mathrm{o}}$ is half the initial dislocations length. This last equation is the condition of line tension equilibrium at the intersection of slip planes of dislocations (1) and (2). On the left side, the equilibrium condition can be written only if we pay attention to a simple trick. The radius of curvature, $R_{\underline{2}}$, is still equal to $\mu b / \tau_{\underline{F}}$ since dislocation (2) is unloaded. But $R_{1}$ on the loaded dislocation becomes close to $\mu b /\left(2 \tau_{F}\right)$, because, now, two resistances oppose to the 
proceeding of the annihilation: the alloy friction $\tau_{F}$ and the applied stress $\tau_{a p p}$, which pushes dislocation (1) towards the initial intersection point, i.e. against the annihilation reaction. These two resistances are equal since the applied stress $\tau_{a p p}$ is taken equal to $\tau_{F}$. By writing

$$
X_{2}=\frac{\sin \left(\beta+\theta_{2}\right)}{\sin (\beta)} \cot \left(\frac{\theta_{2}+\beta}{2}\right) \frac{\mu b}{2 \tau_{F}}=\frac{\sin \left(\theta_{2}-\beta\right)}{\sin (\beta)} \tan \left(\frac{\theta_{2}-\beta}{2}\right) \frac{\mu b}{\tau_{F}}
$$

\section{This equation shows that $\theta_{2}$ is independent of $\tau_{\underline{F}}$ and it can be simplified to obtain $3 \cos \theta_{2}$ $\underline{\cos \beta+\sin \theta_{2}} \underline{\sin \beta=1 \text {, which enables the calculation of } \theta_{2}}$.}

From equations 4 and 5 , it is possible to verify numerically that, when $\tau_{F}$ is large enough, the total annihilation length, $X=X_{1}+X_{2}$, becomes proportional to $\mu b / \tau_{F}$. The difference, with respect to the solution obtained in the unloaded cases expressed in equation 3, comes from the fact that this time the reaction extent, i.e. the annihilation distance and the junction length, depends on the dislocation initial length $2 L_{\mathrm{o}}$. This is important for it suggests that the influence of $\tau_{F}$ will decrease when the forest dislocation spacing decreases. In other terms, since the dislocation density increases during plastic deformation, the effect of alloy friction on dislocation interactions decreases with plastic strain.

\section{Mesoscopic Dislocation Dynamics simulations}

In the previous sections, it was demonstrated with elementary configurations that junction lengths and annihilation distances are modified by solute friction and this effect is almost proportional to the screening distance, $R=\mu b / \tau_{F}$. In this second part, we explore how this 
In FCC crystals, Lomer junctions are commonly considered as the most potent forest reaction in multislip conditions. This is justified for instance by the fact that the amplitude of the coefficient of interaction measured in latent hardening tests involving only Lomer junctions is close to the measured coefficient $\alpha$ in [001] tensile tests. This result has been verified experimentally [15] as well as with DD simulations [14].

(Figure 4)

The two microstructures reproduced in figure 4 are instantaneous pictures taken from DD simulations of latent hardening tests using $\mathrm{Cu}$ as model material $(\mu=42 \mathrm{GPa}, v=0.3$ and $b$ $=0.255 \mathrm{~nm})$. The simulation conditions are the following. Long dislocation lines of a reference slip system are stressed and glide through a relaxed microstructure. The latter is made of a random distribution of forest dislocations constructed under periodic boundary conditions. The Burgers vectors of the forest dislocations are those permitting the formation of Lomer junctions. Both images represent the same thin foil extracted from a larger simulation cell of $(15 \mu \mathrm{m})^{3}$. The initial segment configuration and the loading conditions in both calculations are jdentical. The results are compared at the same level of plastic 
deformation. Hence, the differences between Fig 4-a and 4-b can only result from the different values of $\tau_{F}$ used (i.e. 1 and $30 \mathrm{MPa}$, respectively).

In agreement with the predictions of the previous section, extensive DD simulations involving hundreds of Lomer junctions in the reference volume report a systematic decrease of the junction lengths when the magnitude of $\tau_{F}$ is increased. This effect is clearly shown on the two microstructures reproduced in figure 4, where the Lomer junctions formed at the intersection of dislocation glide planes are highlighted as bold lines. It is important to note that the number of formed junctions also decreases with increasing $\tau_{F}$, resulting in larger spacing between junctions. A strong decrease of the interaction coefficient is therefore expected.

It is in addition useful to analyse how $\alpha_{L}$ depends on $\tau_{F}$. The quantity $\alpha_{L}=\left(\tau_{c}-\tau_{F}\right) /(\mu b \sqrt{\rho})$, can be directly determined from the feed back achieved on $\tau_{a p p}$ during the simulation in order to impose a constant plastic strain rate of $1 \mathrm{~s}^{-1}$. Figure 5 shows the evolution of $\alpha_{L}$ when the friction stress is increased from 0.1 to $50 \mathrm{MPa}$ for a forest density $\rho=10^{12} \mathrm{~m}^{-2}$. Two asymptotic behaviours can be recognized from this figure: at small values of $\tau_{F}, \alpha_{L}$ is constant, when at large values of $\tau_{F}, \alpha_{L}$ decreases proportionally to $\ln \left(\mu b / \tau_{F}\right)$.

(Figure 5)

This clearly confirms that a large friction stress screens elastic interactions at a cut-off distance about $\mu b / \tau_{\mathrm{F}}$.

\section{Discussion}

$-10-$ 
The general relation between $\alpha$ and $\tau_{F}$ is complex and involves collective behaviour which we have been able to reproduce by dislocation dynamics where, for instance, dislocations are commonly observed to glide throughout the forest by bursts corresponding to the cooperative unzipping of several junctions.

The present discussion is based mainly on the results of latent hardening simulations shown in Figure 5, but the reasoning is quite general, and it can be extended to all types of dislocation reactions and all crystallographic structures. There are essentially two fundamental features affected by alloy friction:

(i) The decrease of the junction length, cf. last section, causes the junction arms to remain close to each other. These arms are attractive and the corresponding interaction energy should, therefore, be subtracted from the energetic gain generated by the junction formation. Consequently, the driving force as well as the intrinsic strength of junctions and annihilation reactions are expected to decrease. The distance between attractive arms is roughly proportional to the screening factor $R=\mu b / \tau_{F}$. This attraction decreases the line tension of curved dislocation arms in a manner similar to that described by Bacon et al. [16] for the Orowan mechanism. Under a line tension approach of forest hardening, such an effect is taken into account by a reduction of the outer cut-off radius, $D$, in the logarithmic term of the dislocation line energy. According to Bacon et al. [16], $D$ can be defined as a harmonic average:

$$
D=\frac{R \cdot l}{(R+l)},
$$

where $l$ is the average spacing between pinning junctions. Usually, $l$ is considered to be dependent only on forest density $\left(l \propto \rho_{f}^{-1 / 2}\right)$. In the present work, the forest density is constant,

\section{Deleted: length weakens}

Deleted: reactions, because of the

dipolar attraction of junction arms, which remain close because of the decreasing of junction lengths and annihilation distance, cf. last section. 
but the strength of forest interaction decreases with $\tau_{\mathrm{F}}$. Hence, as suggested by Friedel [17], the number of simultaneous pinning junctions along mobile dislocations decreases when $\tau_{\mathrm{F}}$ increases (cf. figure 4) resulting in noticeable variations of the average junction spacing, $l$. The variations of $l$ were measured in DD simulations and compared with theoretical predictions.

The stability of forest junction decreasing with increasing value of $\tau_{F}$, we hypothesize that the simulation results can be interpreted with the statistical model of Friedel [17] in the case of weak obstacles which stipulates that $l$ varies proportionally to $F^{1 / 2}$, where $F$ is the obstacles strength or equivalently a critical pinning force. Since, in the present study, the variation of the junction strength is related to $R$, the mean junctions strength is expected to vary with $\ln (D / b)$. The evolution of the average junction spacing, $l$, measured by DD simulation and normalized by the average distance of forest dislocation $\rho^{-1 / 2}$, as function of $\ln (D / b)^{-1 / 2}$ is shown in figure 6. The linear tendency is conspicuous, in agreement with the Friedel model.

In addition, figure 6 reveals a strong dependence of $l$ upon $\tau_{\mathrm{F}}$. The latter quantity increases from $1.5 \rho^{-1 / 2}$ to $6 \rho^{-1 / 2}$ when $\tau_{\mathrm{F}}$ is varied from 1 to $50 \mathrm{MPa}$. This relative variation of $l$ is of the same order as the variation of $\alpha$, which implies that increasing $\tau_{\mathrm{F}}$ affects, essentially, the average spacing between junctions.

Another point related to the harmonic average quantity $D$ is suggested by figure 5 and confirmed in figure 6. Two domains should be separated depending on the ratio between $R$, the screening distance, and the average spacing of forest dislocations, $l_{\mathrm{o}}=1 / \sqrt{\rho}$. When $R>$ $l_{o}, D$ is almost equal to $l_{o}$ and the alloy friction does not play any role; the interaction coefficient $\alpha$ is almost the same as $\alpha_{0}$, measured in the pure material. When $l_{o}>R, D$ 
approaches $R=\mu b / \tau_{\mathrm{F}}$. In this case the forest model does not apply and $\alpha$ varies strongly with $\tau_{\mathrm{F}}$. These two domains are clearly emphasized in the two asymptotic behaviours revealed in figure 5 .

(ii) The second important feature suggested by simulations is related to the dislocation curvature between junctions. In a conventional manner, one would assume curvature to be close to the average junction spacing, $l$, weighted by the intrinsic junction strength $\alpha_{\mathrm{o}}[8]$. By combining features (i) and (ii), one can identify from a line tension approach the evolution of the critical stress as:

$$
\tau_{c}-\tau_{F}=\alpha \mu b \sqrt{\rho}=\alpha_{o} A \frac{\mu b}{l} \ln \frac{l}{\alpha_{o} b},
$$

where, $A$ is a geometrical constant accounting for the mean character of dislocations. From equation 7 and the evolution of $l$ reported in figure 6 , a simple relation between $\alpha$ and $\alpha_{0}$ can be derived:

$$
\alpha=\alpha_{o} \frac{A}{l \sqrt{\rho}} \ln \frac{l}{\alpha_{o} b} .
$$

The validity of this relation is tested in figure 7 in the high friction regime $(l>R)$. Clearly, the variations of $\alpha$ are consistent with the above formula, which mathematically accounts for the stress necessary to bow out a dislocation between obstacles with a given radius of curvature. Analysis of simulation results with a regression line provide the value of $\mathrm{A}=0.22$, which is close to $1 /[2 \pi(1-v)]$. This last value is consistent with the fact that the hardest direction of dislocation propagation in the forest density is the screw direction. One can thus express the variation of the interaction coefficient without any adjustable parameter: 


$$
\alpha=\frac{\alpha_{o}}{2 \pi(1-v) l \sqrt{\rho}} \ln \frac{l}{\alpha_{o} b} .
$$

Equation 9 allows us to describe the evolution of the interaction coefficient with friction stress.

\section{Conclusion}

Alloy friction can reduce the range of effective elastic interactions between dislocations. In the present paper we show that it restricts the driving forces involved during forest reactions

in a region scaling with $R=\mu b / \tau_{\mathrm{F}}$. The main consequence is a strong decrease of the number of stable junctions leading to a large spacing between pinning forest dislocations. A model is proposed to relate, without adjustable parameters, the decrease of the interaction coefficient to the modification of the line tension induced by increasing friction stress. In all cases, during plastic deformation, the increase in dislocation density progressively eliminates the contribution of alloy friction to strain hardening.

\section{Acknowledgement}

The authors are grateful to Dr. Ladislas Kubin and Dr. Patrick Veyssière for valuable discussions. This work was partly supported by the European project PERFECT (FI60-CT2003-208840).

$-14-$ 
1

2

3

4

5

6

7

8

9

10

11

12

13

14

15

16

17

18

19

20

21

22

23

24

25

26

27

28

29

30

31

32

33

34

35

36

37

38

39

40

41

42

43

44

45

46

47

48

49

50

51

52

53

54

55

56

57

58

59

60

\section{References}

[1] Mott N.F. and Nabarro F.R.N., Proc. R. Soc., 52, 86 (1940).

[2] Haasen P., "Physical Metallurgy", Cambridge University Press (1996).

[3] Gottstein G., "Physical Foundations of Materials Science", Springer (2004).

[4] Saada G. and Veyssière P., in "Dislocations in Solids" Vol. 11, 413 (2002).

[5] Madec R., Devincre B. and Kubin L.P., Phys. Rev. Lett., 89, 255508 (2002).

[6] Saada G., Acta Met., 8, 841 (1960).

[7] Schoeck G. and Frydman R., Phys. Stat. Sol. (b), 53, 661 (1972).

[8] Wickham L. K., Schwarz K. W. and Stölken, J. S., Phys. Rev. Lett., 83, 4574 (1999).

[9] Madec R., Devincre B. and Kubin L.P., Comp. Mat. Sci., 23, 219 (2002).

[10] Dupuy L. and Fivel M.C., Acta Mater., 50, 4873 (2002).

[11] Devincre B., Kubin L., Lemarchand C. and Madec R., Mater. Sci. Eng. A, 309-310, $211(2001)$.

[12] Monnet G., Devincre B. and Kubin L.P., Acta Mater., 52, 4317 (2004).

[13] Tang M., Kubin L.P. and Canova G., Acta Mater., 46, 3221 (1998).

[14] Madec R., Devincre B., Kubin L., Hoc T. and Rodney D., Science, 301, 1879, (2003).

[15] Franciosi P., Berveiller M. and Zaoui A. Acta metall., 28, 273 (1980).

[16] Bacon D., Kocks U. and Scattergood R., Phil. Mag., 28, 1241 (1973).

[17] Friedel J. "Dislocations”. Pergamon Press, Oxford, (1964). 


\section{Figures:}

Figure 1: Initial (dashed lines) and final (continuous line) shapes of the dislocation arms involved in symmetrical junction between two initially edge dislocations with a strong friction stress.

Figure 2: Evolution of relaxed length of the junctions formed at the intersection between two edge dislocations in $\mathrm{BCC}$ iron as a function of $\mu b / \tau_{F}$. Intersecting dislocations are respectively

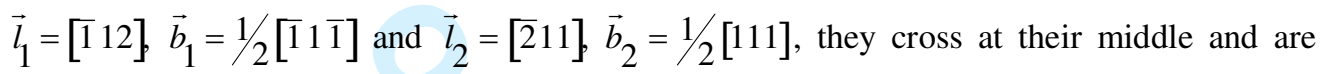
$4 \mu \mathrm{m}$ long. For this simulation we considered $\mu=74 \mathrm{GPa}, v=0.3$ and $b=0.248 \mathrm{~nm}$. Squares denote DD results and the solid line refers to the solution of Eq. (3).

Figure 3: Reproduction of the equilibrium configuration calculated by DD simulation for the case of a symmetrical collinear annihilation, but in crossing glide planes. The Schmid factor is set to zero on system (2) and the applied shear stress on system (1) equals $\tau_{F}$.

Figure 4: Thin foils of $0.2 \mu \mathrm{m}$ thick taken from DD simulations of latent hardening tests. Simulations are using periodic boundary conditions in a reference volume about $(15 \mu \mathrm{m})^{3}$. Gray lines are dislocation of the primary slip system 1/2 [011](111 $)$ cutting a forest density made exclusively of $1 / 2[101](111)$ and $1 / 2[110](111)$ dislocations $\left(\rho_{f} \approx 10^{12} \mathrm{~m}^{-2}\right)$. Bold segments are Lomer junctions. (A) DD simulation with $\tau_{F}=1 \mathrm{MPa}$ and (B) with $\tau_{F}=30 \mathrm{MPa}$.

Figure 5: Variations of the forest coefficient $\alpha_{L}$ calculated in latent hardening test involving only Lomer junction as a function of friction stress in reduced unit $\mu b / \tau_{F}$.

$-16-$ 
Figure 6: Variation of the normalized average dislocation length between junctions, obtained from DD simulations, as a function of the junction strength, according to the Friedel model [17].

Figure 7: relative variation of the interaction coefficient as a function of the modification of the line tension variation. 


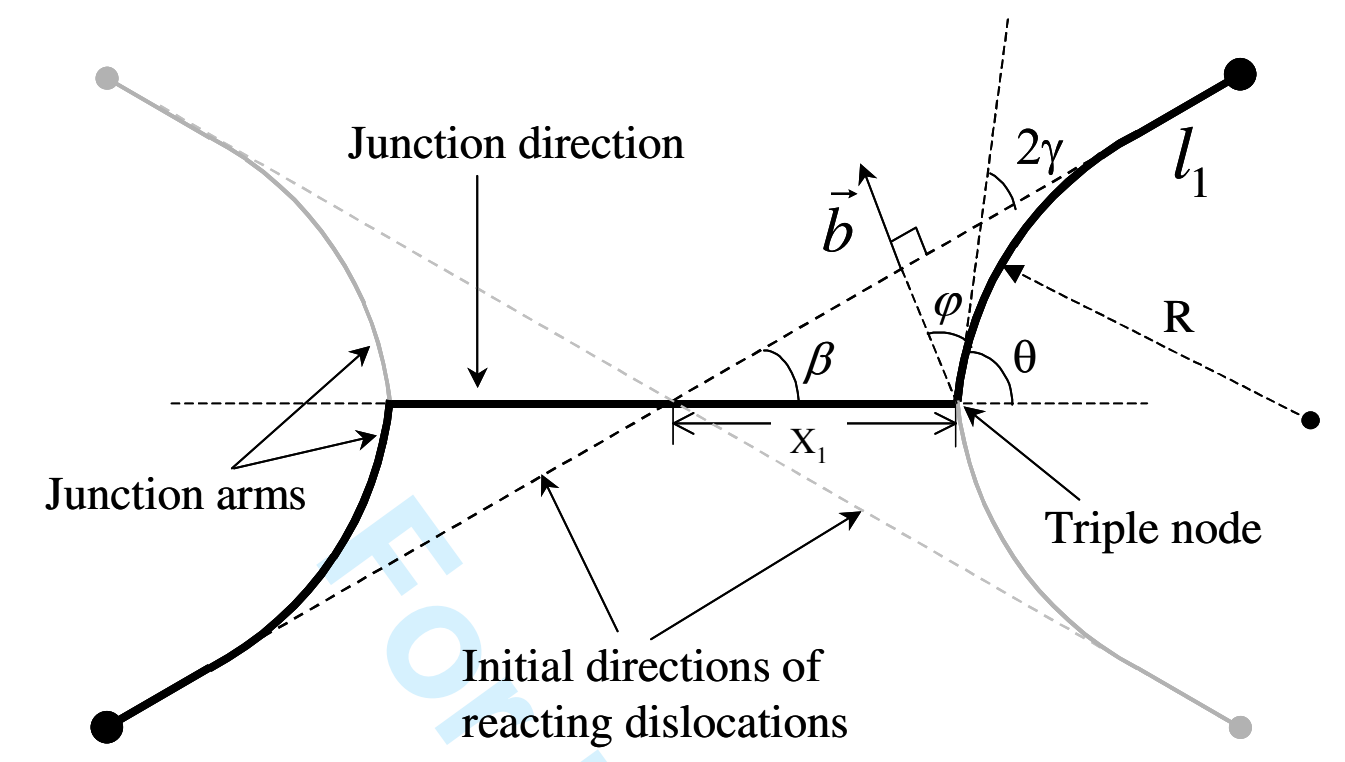

\section{(Figure 1)}

Initial dislocation direction

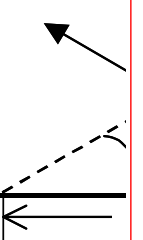




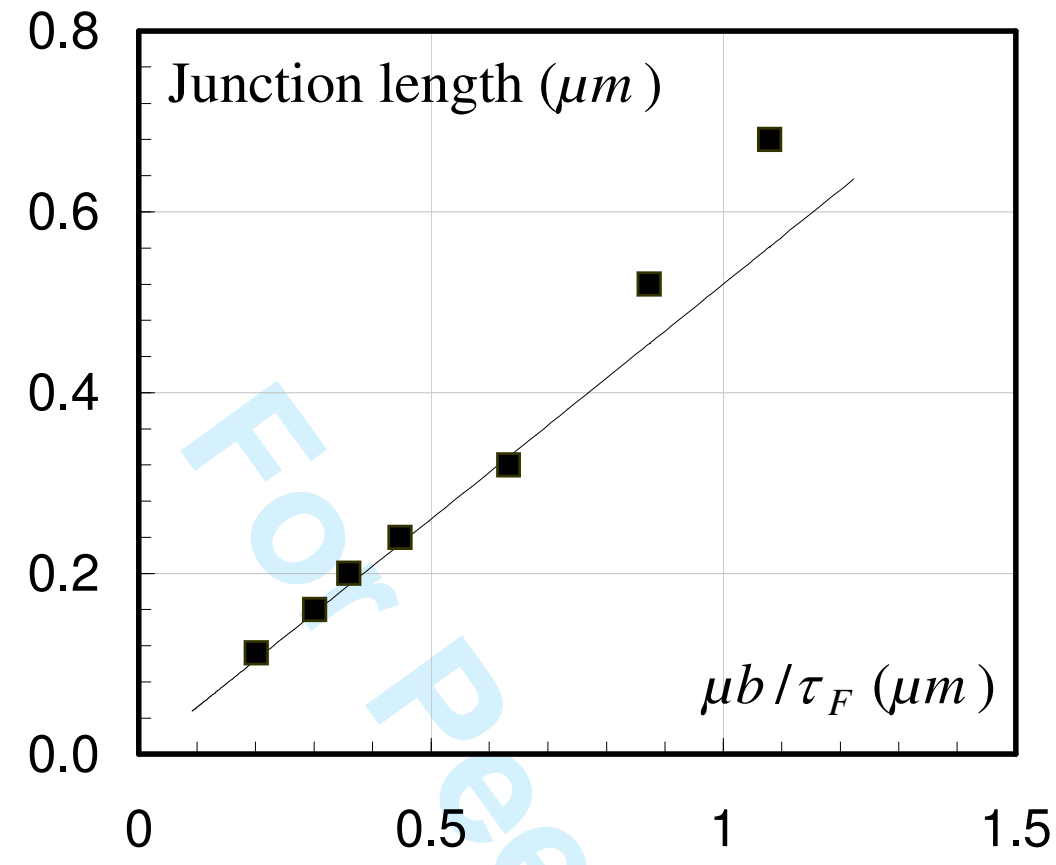

(Figure 2)

$-19-$ 


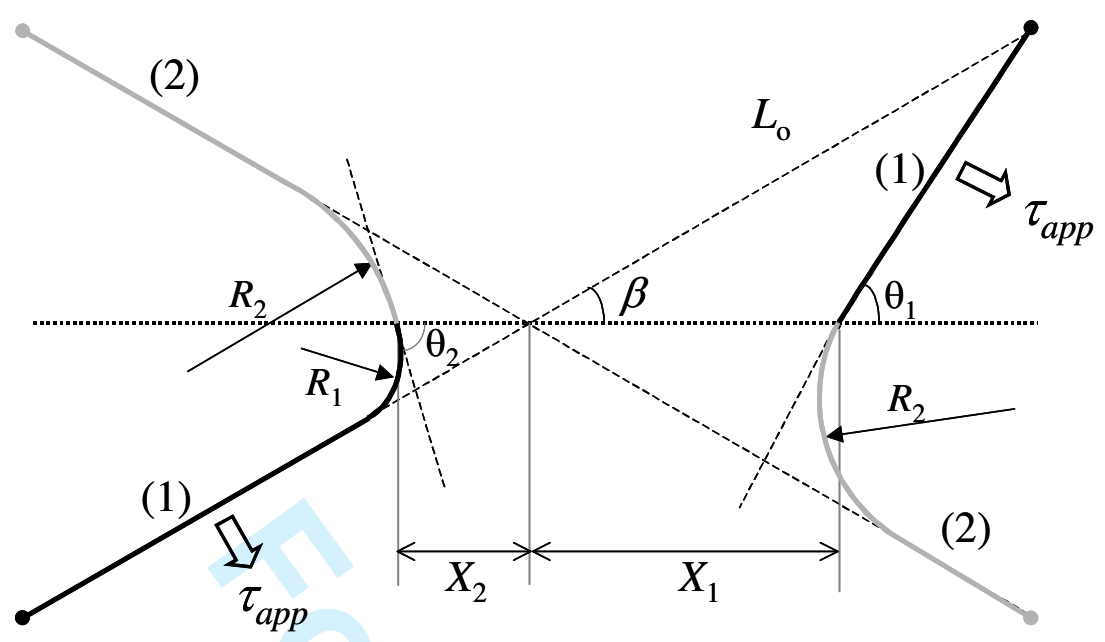

(Figure 3) 


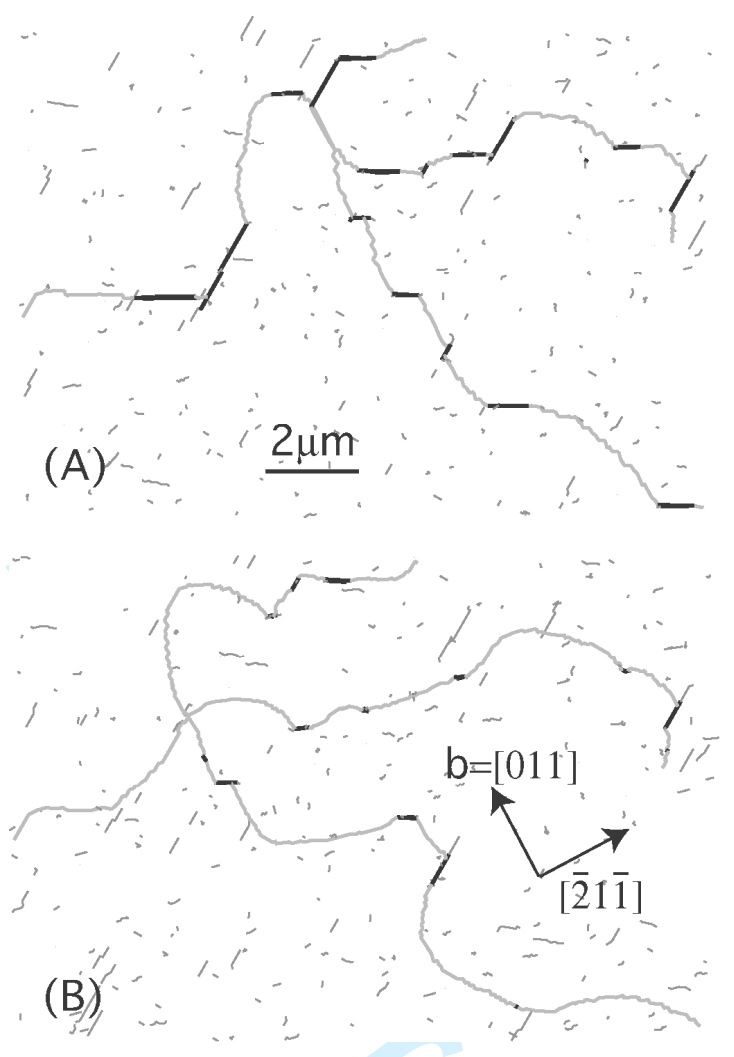

(Figure 4) 


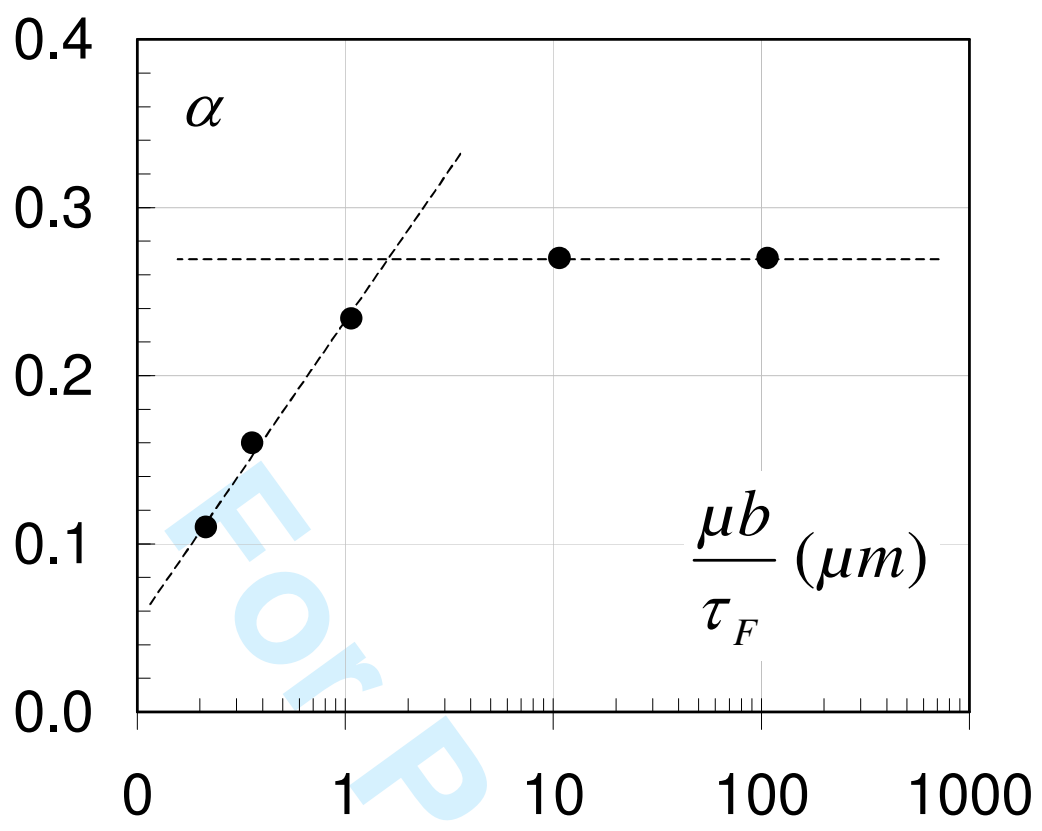

(Figure 5)

$-22-$ 


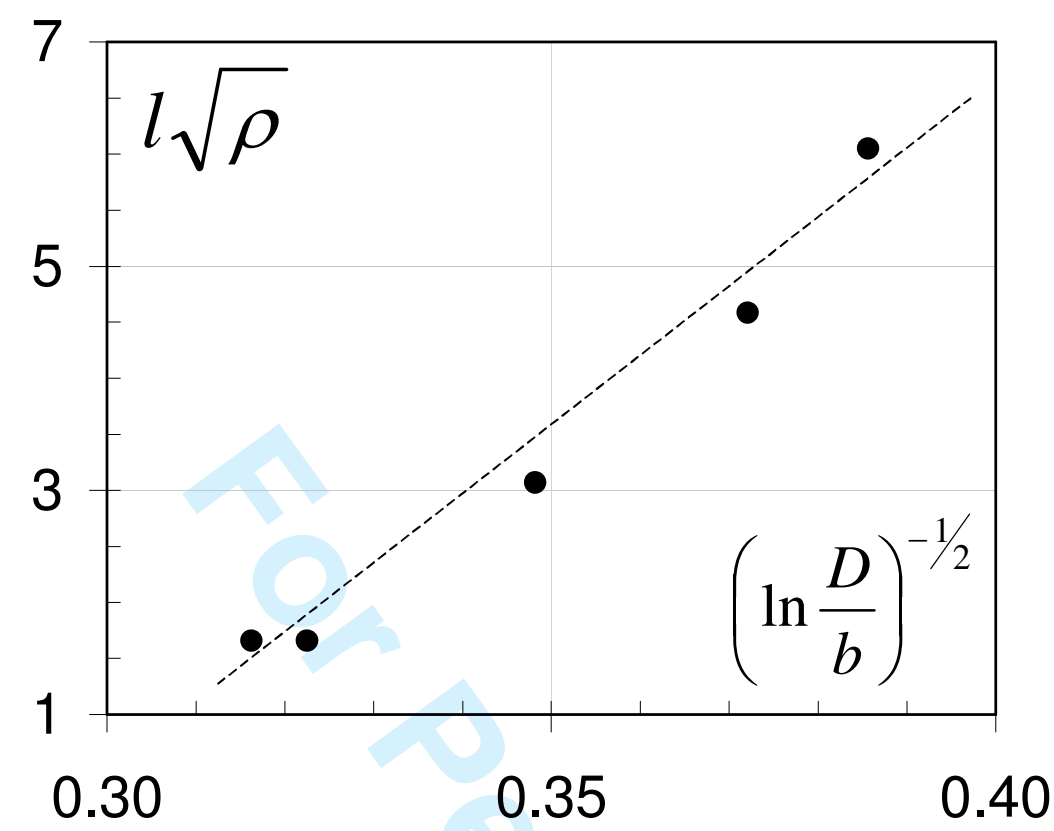

(Figure 6)

$-23-$ 


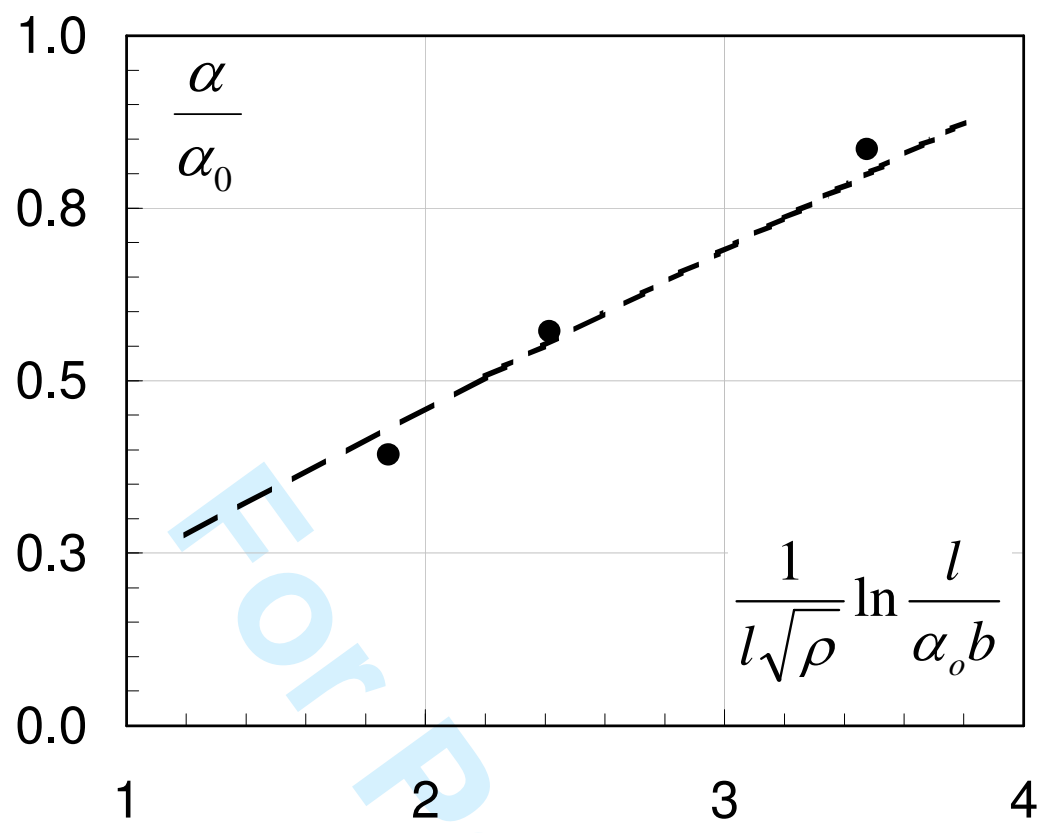

(Figure 7) 